CASE REPORT

\title{
Leiomyoma of the Vulva
}

\section{Nagat Bettamer $^{1^{*}}$, Ream Langhe ${ }^{1^{*}}$, Zahia Elghazal ${ }^{1}$, Farag Ben Ali ${ }^{1}$ and Lamia Alkikhia ${ }^{2}$}

${ }^{1}$ Department of Obstetrics and Gynaecology, University of Benghazi, Benghazi, Libya

${ }^{2}$ Department of Pathology, University of Benghazi, Benghazi, Libya

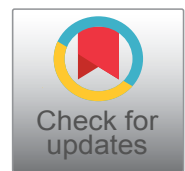

*Corresponding author: Nagat Bettamer and Ream Langhe, Department of Obstetrics and Gynaecology, University of Benghazi, Benghazi, Libya

\begin{abstract}
The authors present a rare case of vulval swelling in a 2-year period in a 35-year-old woman. The swelling was associated with mild vulval discomfort but no other symptoms. The tumour was removed surgically with no complications. Histopathology of the swelling confirmed vulval leiomyoma. Postoperative recovery was unremarkable and the woman was discharged on postoperative day 2 .
\end{abstract}

\section{Keywords}

Leiomyoma, Swelling, Vulva

\section{Case Presentation}

A 35-year-old patient presented to our department with a history of painless left vulval swelling of over a duration of two years. The three previous uncomplicated vaginal deliveries and her last delivery was 3 years ago. The woman was swelling was slowly growing and

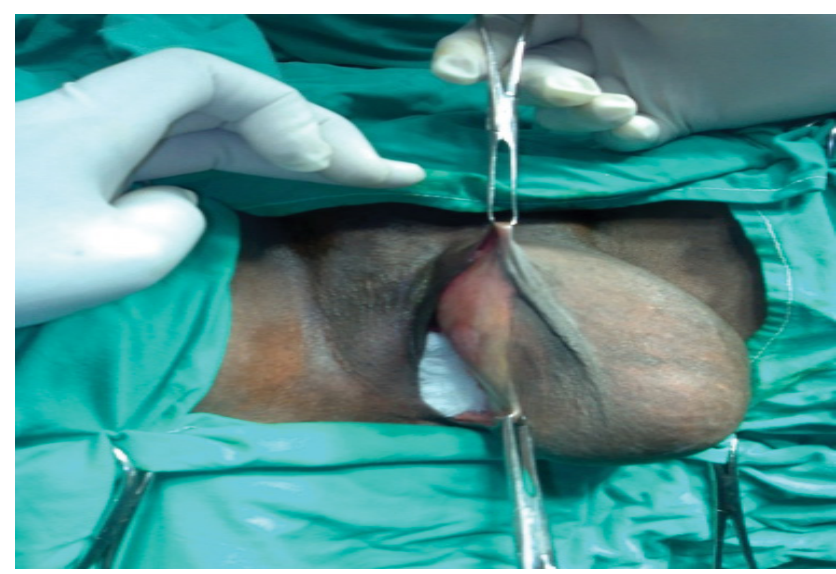

Figure 1: Left vulval swelling. associated with mild discomfort but no other symptoms. The woman had non-smoker and of a normal BMI. Her medical and surgical histories were unremarkable. There was no family history of any malignancies.

On examination, the the patient was well, and her vitals were stable. Examination of her breasts and abdomen was unremarkable.

\section{Local examination}

Showed enlarged left side vulval swelling $12 \times 10 \mathrm{~cm}$, firm, fairly mobile with no tenderness and no signs of inflammation (Figure 1). The uterus was of normal size and there was no adnexal mass.

Abdominal, transvaginal and transperineal scan revealed a cystic swelling in the left vulva with degenerative changes. No other pathology was detected in the scan.

The woman was counselled and consented for surgical excision under general anesthesia. At surgery, a vertical incision was performed on the inner side of the left labium majus. The tumour was inoculated and excised. Homeostasis was secured and the tissues sent for histopathology (Figures 2a and Figure 2b).

\section{Histopathology Report}

Gross examination revealed a well circumscribed non encapsulated soft tissue firm mass $12 \times 11 \times 13 \mathrm{~cm}$ size grey whorled with yellow spots.

\section{Microscopy}

The section revealed a benign neoplasm composed of interlacing bundles of spindled cells with abundant 

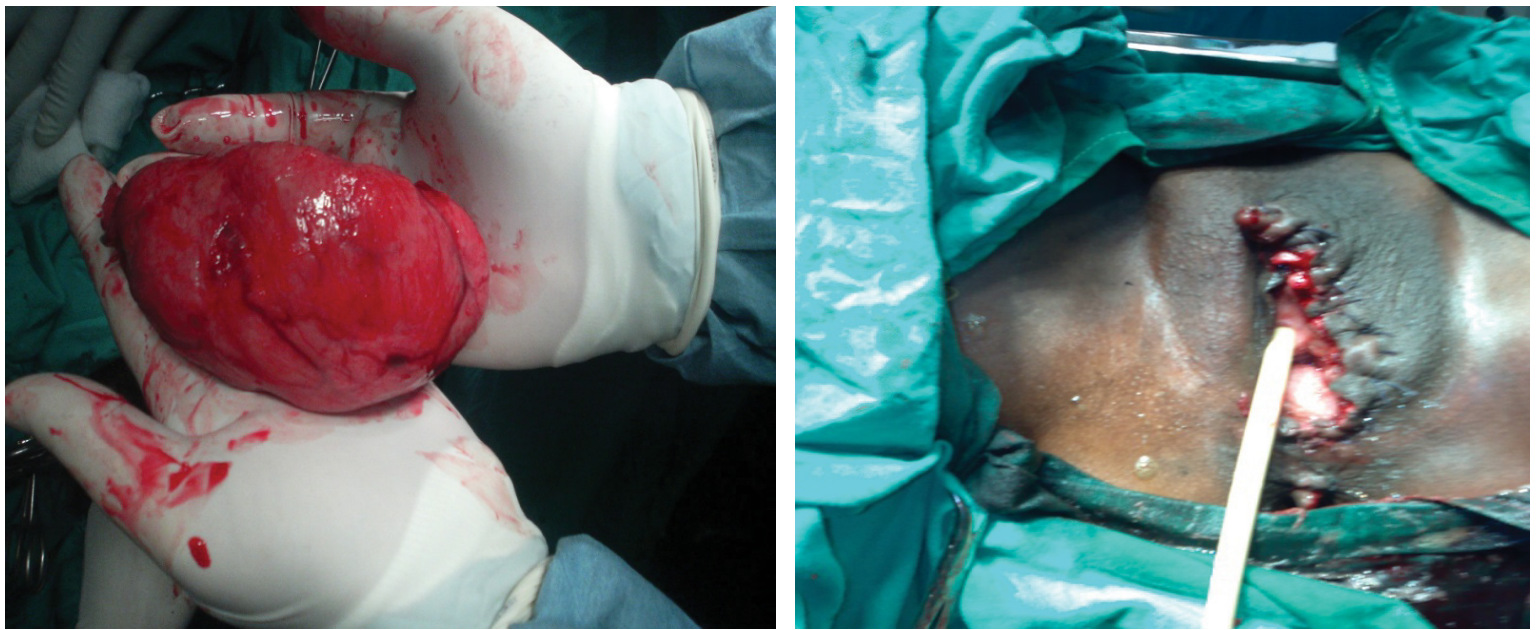

Figure 2: (a) Shows a swelling removed from left vulva; (b) Illustrates vulva appearance after removal of swelling.

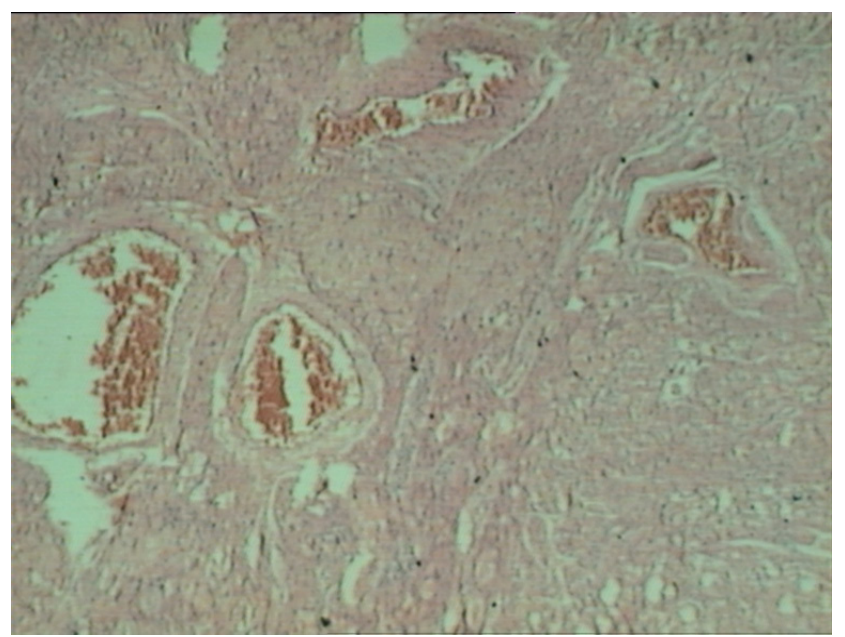

Figure 3: Illustrates smoth muscle fibres with blood vessels.

eosinophlic cytoplasm and delicate fibrovasculr stroma with areas of many blood vessels, no malignancy (Figure $3)$. The diagnosis confirmed vulval leiomyoma.

\section{Discussion}

Vulvar leiomyoma is a rare type of benign tumours of smooth muscle cells. So far, about 160 cases of vulval leiomyoma have been reported in English literature [14].

Vulvar leiomyomas occur during the reproductive years and most patients present with a painless nodule or swelling in the labia. This benign tumour usually remains small for long period of time and grows slowly in the early period. As the swelling increases in size patients started to experience symptoms such as pain, itching and erythema $[5,6]$. The patient in index was asymptomatic for 2 years and she did not seek any medical advice until she started to experience discomfort.

Differential diagnosis of vulval leiomyoma includes urBartholin cyst, Bartholin abscess, fibromas, lymphangiomas, soft-tissue sarcomas, and neurogenic tumos [5]. Differentiation between benign and malignant vul- val lesions can be challenging. This is due to rarity of the lesions and non-specific clinical presentation [7]. On examination, vulval leiomyoma are usually non-tender, mobile with firm consistency [8-11].

Ultrasound is the most reliable imaging tool in establishing the diagnosis of uterine and extrauterine leiomyoma [5,12]. Magnetic resonance imaging (MRI) is useful in differentiating benign and malignant lesions in difficult cases $[4,5]$. A characteristic feature of malignant lesions on MRI is low signal intensity on T2-weighted images [1].

Surgical excision of the tumour along with some of the surrounding tissues is the treatment of choice of vulval leiomyoma $[1,3,4]$. Histologically, leiomyomas of the vulva are generally similar to their more commonly occurring counterparts in the uterine body. Follow up after surgery is recommended because of risk of recurrence.

\section{Conclusion}

Vulval leiomyoma is a rare type of benign tumour in the vulva. This condition is often misdiagnosed as Bartholin cyst. Transperineal ultrasound helps in establishing the diagnosis. Surgical excision is the best current treatment available and histopathology confirmed the diagnosis. Postoperative follow up is highly recommended for these patients.

\section{References}

1. Fasih N, Prasad Shanbhogue AK, Macdonald DB, Fraser-Hill MA, Papadatos D, et al. (2008) Leiomyomas beyond the uterus: Unusual locations, rare manifestations. Radiographics 28: 1931-1948.

2. Reyad MM, Gazvani MR, Khine MM (2006) A rare case of primary leiomyoma of the vulva. Journal of obstetrics and gynaecology 26: 73-74.

3. Zhao T, Liu X, Lu Y (2015) Myxoid epithelial leiomyoma of the vulva: A case report and literature review. Case Rep Obstet Gynecol.

4. Kurdi S, Arafat AS, Almegbel M, Aladham M (2016) Leio- 
myoma of the Vulva: A diagnostic challenge case report. Case Reports in Obstetrics and Gynecology.

5. Pandey D, Shetty J, Saxena A, Srilatha PS (2014) Leiomyoma in vulva: A diagnostic dilemma. Case Reports in Obstetrics and Gynecology.

6. Nielsen GP, Rosenberg AE, Koerner FC, Young RH, Scully RE (1996) Smooth-muscle tumors of the vulva: A clinicopathological study of 25 cases and review of the literature. The American Journal of Surgical Pathology 20: 779-793.

7. Safaa A, Chourouk E, Najia Z, Amina L, Abdelaziz B (2019) Vulvar leiomyoma: A case report. Pan Afr Med J.

8. Sun C, Zou J, Wang Q, Wang Q, Han L, et al. (2018) Review of the pathophysiology, diagnosis, and therapy of vulvar leiomyoma, a rare gynecological tumor. J Int Med Res 46: 663-674.
9. Roy KK, Mittal S, Kriplani A (1998) A rare case of vulval and perineal leiomyoma. Acta Obstetricia et Gynecologica Scandinavica 77 : 356-357.

10. Kransdorf MJ, Meis-Kindblom JM (1994) Dermatofibrosarcoma protuberans: Radiologic appearance. AJR. American Journal of Roentgenology 163: 391-394.

11. Ngo Q, Haertsch $P$ (2011) Vulvar leiomyoma in association with gastrointestinal leiomyoma. The Australian \& New Zealand Journal of Obstetrics \& Gynaecology 51: 468.

12. Tavares KA, Moscovitz T, Tcherniakovsky M, Pompei LD, Fernandes CE (2017) Differential diagnosis between Bartholin cyst and vulvar leiomyoma: Case report. Revista Brasileira de Ginecologia e Obstetricia. 39: 433-435. 